\title{
Density functional theory for molecular multiphoton ionization in the perturbative regime
}

Daniele Toffoli, and Piero Decleva

Citation: The Journal of Chemical Physics 137, 134103 (2012);

View online: https://doi.org/10.1063/1.4754820

View Table of Contents: http://aip.scitation.org/toc/jcp/137/13

Published by the American Institute of Physics

\section{Articles you may be interested in}

Time-dependent density-functional theory for molecular photoionization with noniterative algorithm and multicenter $B$-spline basis set: $C S_{2}$ and $C_{6} H_{6}$ case studies

The Journal of Chemical Physics 122, 234301 (2005); 10.1063/1.1937367

\section{AIP $\mid$ The Jounal of Chemical Physics}




\title{
Density functional theory for molecular multiphoton ionization in the perturbative regime
}

\author{
Daniele Toffoli ${ }^{1, a)}$ and Piero Decleva ${ }^{2}$ \\ ${ }^{1}$ Department of Chemistry, Middle East Technical University, 06531 Ankara, Turkey \\ ${ }^{2}$ Dipartimento di Scienze Chimiche e Farmaceutiche, Università degli Studi di Trieste, \\ Via L. Giorgieri 1, I-34127, Trieste, Italy and CNR-IOM DEMOCRITOS, Trieste, Italy
}

(Received 26 July 2012; accepted 12 September 2012; published online 2 October 2012)

\begin{abstract}
A general implementation of the lowest nonvanishing order perturbation theory for the calculation of molecular multiphoton ionization cross sections is proposed in the framework of density functional theory. Bound and scattering wave functions are expanded in a multicentric basis set and advantage is taken of the full molecular point group symmetry, thus enabling the application of the formalism to medium-size molecules. Multiphoton ionization cross sections and angular asymmetry parameters have been calculated for the two- and four-photon ionization of the $\mathrm{H}_{2}^{+}$molecule, for linear and circular light polarizations. Both fixed and random orientations of the target molecule have been considered. To demonstrate the efficiency of the proposed methodology, the two-photon cross section and angular asymmetry parameters for the HOMO and HOMO-1 orbital ionization of benzene are also presented. () 2012 American Institute of Physics. [http://dx.doi.org/10.1063/1.4754820]
\end{abstract}

\section{INTRODUCTION}

The theoretical treatment of multiphoton (MP) ionization processes, in the framework of lowest order perturbation theory (LOPT), has been available for more than three decades for atomic systems. ${ }^{1-3}$ Progress in the molecular case has been much slower, despite few pioneering studies by Cacelli et al. ${ }^{4,5}$ on hydrides. The paper of Boeglin et al. ${ }^{6}$ detailing the development and application of the density matrix method and a recent contribution by Son and Chu on the multiphoton ionization of $\mathrm{N}_{2}$ and $\mathrm{H}_{2} \mathrm{O}$ by means of a grid-based time dependent density functional theory method, ${ }^{7}$ are worth mentioning. In particular, $\mathrm{H}_{2}^{+}$and $\mathrm{H}_{2}$ have been the workbench for theoretical studies and have been repeatedly investigated in great detail. ${ }^{2,8-13}$ Also from the experimental side not much work has been devoted to molecular ionization in the nonresonant regime, although the resonantly enhanced multiphoton ionization (REMPI) technique has become a very popular spectroscopic as well as an analytical tool. ${ }^{14}$ This is in part motivated by the steadfast increase in laser power, which has quickly pushed the main experimental emphasis in the nonperturbative, strong field regime. In particular the possibility of obtaining extremely short pulses, now in the attosecond domain, opens the possibility of exploring nuclear as well as electron dynamics in atoms and molecules, with fascinating perspectives. ${ }^{15}$

The recent development of the free electron laser (FEL), pioneered at FLASH, ${ }^{16}$ with a bunch of new facilities just having entered operation or under construction, changes somewhat the situation, reviving the multiphoton regime. In fact, despite the enormous powers available, because of the much larger photon energies, the ponderomotive energy $U_{p}$ is tiny, so that the radiation-matter interaction is well inside the MP

\footnotetext{
a) Author to whom correspondence should be addressed. Electronic mail: dtoffoli@metu.edu.tr.
}

domain. The same holds also for the high harmonic generation sources. Also, except for the shortest pulses, pulse duration generally exceeds a few tens of optical cycles, making the LOPT approach completely justified. ${ }^{17}$

We have then considered interesting to expand the molecular approach and code developed over the years, ${ }^{18-20}$ which has been very successful in the study of one photon molecular ionization processes, to the evaluation of MP ionization cross sections. The use of a multicenter B-spline basis offers the capability of attaining convergent results, within the chosen Hamiltonian model, for photoionization observables also in pretty large molecules, like $\mathrm{C}_{60},{ }^{21}$ camphor, ${ }^{22}$ and $\mathrm{Co}\left(\mathrm{C}_{4} \mathrm{O}_{3} \mathrm{H}_{6}\right)_{3} \cdot{ }^{23}$ On the other hand, the use of B-splines ${ }^{24}$ has become a powerful tool in the calculation of MP and strong field phenomena, both with LOPT and the explicit solution of the time dependent Schrödinger equation (TDSE) ${ }^{25}$ Actually the potential of the present molecular approach, in conjunction with the single electron approximation (SAE) model, in full TDSE calculations has been already demonstrated, with significant success. ${ }^{26}$ It can then be expected that the use of the available machinery can afford the same success in the LOPT regime, and offer therefore the possibility of a first theoretical description for a vast array of experimental situations that may become explored in the immediate future, even for the largest molecular systems. The proposed implementation is completely general, making full use of the molecular point group symmetry. Moreover in this work, the formalism has been implemented so that any field orientation with respect to the molecular frame can be treated, as well as rotational averages for randomly oriented molecules, for both linear or circular light polarizations.

Our first implementation employs a fixed density functional (DFT) Hamiltonian, ${ }^{27}$ therefore in the same spirit of a static exchange, or single channel approach. Already at this level a quite good description of photoionization in the linear regime is generally obtained, and it can be reasonably 
expected that a similar agreement, at least at a semiquantitative level, can be obtained for MP ionization processes. This was indeed observed in the comparison of single channel versus RPA calculations in water. ${ }^{4}$ The basic structure, however, can be in principle extended to a more refined treatment of the many-body problem, time-dependent DFT being an obvious possibility, ${ }^{28,29}$ as well as the use of close-coupling or other ab initio approaches.

The plan of this paper is as follows. First, a review of the basic theory and expressions for the angle resolved multiphoton ionization cross section in the case of a single radiation beam is presented, including angular average for the case of randomly oriented molecules. Second, a brief description of the computational algorithm is presented. An application to the $\mathrm{H}_{2}^{+}$system is then considered, in order to compare with the existing literature, and adding a few results apparently hitherto not considered, like the ionization parameters for the randomly oriented molecule or results for circular polarization. Finally, to show the potential of the approach, results for the ionization of the HOMO and HOMO-1 orbitals of the benzene molecule are presented.

\section{THEORY}

Within the LOPT, the generalized differential cross section for a $N$-photon ionization from an initial bound state $|i\rangle$ to a final state $|f\rangle$ is given by ${ }^{3}$

$$
\frac{d \sigma_{i \rightarrow f}^{(N)}}{d \hat{k}}=2 \pi(2 \pi \alpha \omega)^{N} a_{0}^{2 N} \tau_{0}^{N-1}\left|M_{i \rightarrow f}^{(N)}\right|^{2},
$$

in units of $\mathrm{cm}^{2 N} \mathrm{~s}^{N-1}$. In Eq. (1), $\alpha$ is the fine-structure constant, $a_{0}$ is the atomic unit of length in $\mathrm{cm}$, and $\tau_{0}$ is the atomic unit of time in s; $\omega$ and $M_{i \rightarrow f}^{(N)}$ are, respectively, the photon energy and the multiphoton transition matrix element expressed in atomic units. $M_{i \rightarrow f}^{(N)}$ can be written as

$$
\begin{aligned}
M_{i \rightarrow f}^{(N)}= & \langle f| D_{m_{r}} G\left[\omega_{i}+(n-1) \omega\right] D_{m_{r}} G\left[\omega_{i}+(n-2) \omega\right] \cdots \\
& \times D_{m_{r}} G\left(\omega_{i}+\omega\right) D_{m_{r}}|i\rangle,
\end{aligned}
$$

where in Eq. (2) the continuum state $|f\rangle$ is normalized on the energy scale, $\omega$ is the photon energy, $\omega_{i}$ is the energy of the initial state, and $G(z)=(z I-H)^{-1}$ is the resolvent. $D_{m_{r}}$ is given by

$$
D_{m_{r}}=\hat{\xi}_{m_{r}} \cdot \mathbf{D} \text {. }
$$

In Eq. (3), $\hat{\xi}_{m_{r}}$ is spherical unit vector, specifying the polarization of the radiation in the laboratory frame (LF). The LF $Z$ axis is taken as the direction of polarization of the incident radiation $\left(m_{r}=0\right)$ or the photon propagation axis for circularly polarized light $\left(m_{r}= \pm 1\right)$. $\mathbf{D}$ is the dipole operator in the length gauge. In the representation given by the complete set of the eigenvectors of the molecular Hamiltonian, the transition matrix element $M_{i \rightarrow f}^{(N)}$ is written in terms of $(N-1)$ fold summations over the set of molecular states (bound and scattering states). ${ }^{3}$

In the following, we will derive expressions for the angular distributions of photoelectrons within the fixed nuclei approximation, and for random molecular orientations. For simplicity, we will consider the $N=2$ case, i.e., the two-photon ionization. General expressions for the case of fixed molecular orientation and for $N>2$ can be derived along similar lines in a lengthy but straightforward way.

Following the general treatment of Chandra, ${ }^{30}$ we write the scattering wave function as

$$
|f\rangle \equiv\left|\Psi_{k}^{-}\right\rangle=\sum_{p \mu l h} i^{l} e^{-i \sigma_{l}} X_{l h}^{p \mu *}(\hat{k})\left|\Psi_{l h}^{p \mu-}\right\rangle,
$$

where in Eq. (4) $\sigma_{l}$ is the Coulomb phase shift, $\Psi_{l h}^{p \mu-}$ are $S$ matrix normalized channel functions, and $\hat{k}$ is the direction of the photoelectron momentum in the molecular frame (MF). The $X_{l h}^{p \mu}$ s are symmetry adapted angular functions that transform as the $\mu$ th element of the $p$ th irreducible representation (IR) of the molecular point group. These functions are obtained through a unitary transformation with coefficients $b_{l h m}^{p \mu}$ such that

$$
X_{l h}^{p \mu}(\hat{r})=\sum_{m} b_{l h m}^{p \mu} Y_{l m}(\hat{r}) .
$$

Inserting the expression for the final state wave function into the general expression for the two-photons differential cross section, we then obtain (atomic units are used)

$$
\begin{aligned}
\frac{d \sigma^{(2)}}{d \mathcal{R} d \hat{k}}= & 2 \pi(2 \pi \alpha \omega)^{2} \sum_{\substack{p \mu l h m \lambda_{1} \lambda_{2} \\
p^{\prime} \mu^{\prime} l^{\prime} h^{\prime} m^{\prime} \lambda_{1}^{\prime} \lambda_{2}^{\prime}}} \\
& \times(-i)^{l-l^{\prime}} e^{i\left(\sigma_{l}-\sigma_{l^{\prime}}\right)} b_{l h m}^{p \mu} b_{l^{\prime} h^{\prime} m^{\prime}}^{p^{\prime}{ }^{\prime} *} Y_{l m}(\hat{k}) Y_{l^{\prime} m^{\prime}}^{*}(\hat{k}) \\
& D_{\lambda_{1}, m_{r}}^{(1)}(\mathcal{R}) D_{\lambda_{2}, m_{r}}^{(1)}(\mathcal{R}) D_{\lambda_{1}^{\prime}, m_{r}}^{(1) *}(\mathcal{R}) D_{\lambda_{2}^{\prime}, m_{r}}^{(1) *}(\mathcal{R}) \\
& \times M_{l h}^{p \mu(-)}\left(\lambda_{1}, \lambda_{2}\right) M_{l^{\prime} h^{\prime}}^{p^{\prime} \mu^{\prime}(-) *}\left(\lambda_{1}^{\prime}, \lambda_{2}^{\prime}\right),
\end{aligned}
$$

where the transition matrix elements, $M_{l h}^{p \mu(-)}\left(\lambda_{1}, \lambda_{2}\right)$ are given by

$$
M_{l h}^{p \mu(-)}\left(\lambda_{1}, \lambda_{2}\right)=\sum_{n} \frac{\left\langle\Psi_{l h}^{p \mu-}\left|\hat{\xi}_{\lambda_{1}} \cdot \mathbf{D}\right| n\right\rangle\left\langle n\left|\hat{\xi}_{\lambda_{2}} \cdot \mathbf{D}\right| i\right\rangle}{\omega_{n}-\omega_{i}-\omega} .
$$

In deriving Eq. (6), we have expressed the unit vector of Eq. (3) in the molecular frame of reference

$$
\hat{\xi}_{m_{r}}=\sum_{\lambda} \hat{\xi}_{\lambda} D_{\lambda m_{r}}^{(1)}(\mathcal{R}),
$$

where the argument of the rotation matrix elements, $\mathcal{R} \equiv(\alpha, \beta, \gamma)$, denotes the Euler angles that specify the orientation of the molecular frame of reference with respect to the laboratory fixed frame. If we express the direction of the photoelectron in the laboratory frame of reference by using the relation

$$
Y_{l m}(\hat{k})=\sum_{m^{\prime}} Y_{l m^{\prime}}\left(\hat{k}^{\prime}\right) D_{m m^{\prime}}^{(l) *}(\mathcal{R})
$$

and perform a rotational average we arrive, after a lengthy but straightforward algebra, at the well known expression

$$
\frac{d \sigma^{(2)}}{d \hat{k}^{\prime}}=\frac{\sigma^{(2)}}{4 \pi}\left[1+\sum_{L} \beta_{L} P_{L}(\cos \theta)\right],
$$

where the sum is restricted to $L \leq 4, P_{L}$ are the Legendre polynomials of degree $L$ and $\theta$ is the angle between the direction of the photoelectron momentum vector and the polarization 
vector (propagation direction) for linearly (circularly) polarized photons. In Eq. (10), the angular asymmetry parameters $\beta_{L}=\frac{A_{L}}{A_{0}}$, with $A_{0}=\frac{\sigma^{(2)}}{4 \pi}, \sigma^{(2)}$ being the total cross section. The coefficients $A_{L}$ are given by

$$
\begin{aligned}
A_{L}= & 2 \pi(2 \pi \alpha \omega)^{2}\left(\frac{2 L+1}{4 \pi}\right) \sum_{\begin{array}{c}
p \mu l h m \lambda_{1} \lambda_{2} \\
p^{\prime} \mu^{\prime} l^{\prime} h^{\prime} m^{\prime} \lambda_{1}^{\prime} \lambda_{2}^{\prime}
\end{array}}(-i)^{l-l^{\prime}} e^{i\left(\sigma_{l}-\sigma_{l^{\prime}}\right)} b_{l h m}^{p \mu} b_{l^{\prime} h^{\prime} m^{\prime}}^{p^{\prime} \mu_{J_{2} J_{3}}}(-)^{m+\lambda_{1}^{\prime}+\lambda_{2}^{\prime}} \sqrt{(2 l+1)\left(2 l^{\prime}+1\right)} \\
& \times\left(2 J_{2}+1\right)\left(2 J_{3}+1\right)\left(\begin{array}{ccc}
l & l^{\prime} & L \\
0 & 0 & 0
\end{array}\right)\left(\begin{array}{ccc}
l & l^{\prime} & L \\
-m & m^{\prime} & M
\end{array}\right)\left(\begin{array}{ccc}
1 & 1 & J_{2} \\
\lambda_{1} & -\lambda_{1}^{\prime} & M_{2}
\end{array}\right)\left(\begin{array}{ccc}
1 & 1 & J_{2} \\
m_{r} & -m_{r} & 0
\end{array}\right) \\
& \times\left(\begin{array}{ccc}
1 & 1 & J_{3} \\
\lambda_{2} & -\lambda_{2}^{\prime} & M_{3}
\end{array}\right)\left(\begin{array}{ccc}
1 & 1 & J_{3} \\
m_{r} & -m_{r} & 0
\end{array}\right)\left(\begin{array}{ccc}
J_{2} & J_{3} & L \\
M_{2} & M_{3} & M
\end{array}\right)\left(\begin{array}{ccc}
J_{2} & J_{3} & L \\
0 & 0 & 0
\end{array}\right) \\
& \times M_{l h}^{p \mu(-)}\left(\lambda_{1}, \lambda_{2}\right) M_{l^{\prime} h^{\prime}}^{p^{\prime} \mu^{\prime}(-) *}\left(\lambda_{1}^{\prime}, \lambda_{2}^{\prime}\right)
\end{aligned}
$$

with $m+\lambda_{1}^{\prime}+\lambda_{2}^{\prime}=m^{\prime}+\lambda_{1}+\lambda_{2}$.

\section{IMPLEMENTATION}

The LOPT formalism has been implemented in the multicenter B-spline linear combination of atomic orbitals (LCAO) code, developed by the group. ${ }^{18-20}$ In the DFT framework, the scattering potential is written in term of the ground state density, $\rho(\vec{r})$, and is separated into direct (Hartree), $V_{H}$ and exchange-correlation, $V_{x c}$ terms

$$
h_{K S} \varphi_{i}=\epsilon_{i} \varphi_{i}
$$

with

$$
h_{K S}=-\frac{1}{2} \nabla^{2}-\sum_{i=1}^{N} \frac{Z_{i}}{\left|\vec{r}-\vec{R}_{i}\right|}+\int \frac{\rho\left(\vec{r}^{\prime}\right)}{\left|\vec{r}-\vec{r}^{\prime}\right|} d \overrightarrow{r^{\prime}}+V_{x c}[\rho(\vec{r})] \text {. }
$$

The interaction potential is expanded in a composite basis set, consisting in a large single center expansion (SCE) located at a chosen origin (usually the center of mass of the molecule)

$$
\chi_{n l h}^{p \mu, S C E}=\frac{1}{r} B_{n}(r) \sum_{m} b_{l m h}^{p \mu} Y_{l m}^{R}(\theta, \phi) \equiv \frac{1}{r} B_{n}(r) X_{l h}^{p \mu}(\theta, \phi),
$$

and supplemented by functions of the same type, located on the off-center arbitrary positions $j$,

$$
\chi_{n l h}^{p \mu, i}=\sum_{j \in Q_{i}} \frac{1}{r_{j}} B_{n}\left(r_{j}\right) \sum_{m} b_{l m h, j}^{p \mu} Y_{l m}^{R}\left(\theta_{j}, \phi_{j}\right) .
$$

In Eq. (15), index $i$ runs over the non-equivalent nuclei, $j$ runs over the set of equivalent nuclei, $Q_{i}$, and gives the origin of the off-center spherical coordinates $\left(r_{j}, \theta_{j}, \phi_{j}\right) . B_{n}$ is the $n$th B-spline one-dimensional function ${ }^{24}$ and $Y_{l m}^{R}$ are real spherical harmonics. The B-splines are built over the radial interval $\left[0, R_{\max }^{S C E}\right]$ for the set $\chi_{n l h}^{p \mu, S C E}$, and over the intervals $\left[0, R_{\max }^{i}\right]$ for the off-center functions $\chi_{n l h}^{p \mu, i}$.

With this basis set, Eq. (12) is recast into an algebraic eigenvalue problem, and bound state solutions (orbitals $\varphi_{i}$ ) are obtained with standard generalized diagonalization of the Hamiltonian matrix, whereas scattering states are extracted as the set of linearly independent eigenvectors of the energydependent matrix $A^{\dagger} A$,

$$
A^{\dagger} A(E) c=a c
$$

corresponding to minimum modulus eigenvalues. ${ }^{31}$ In Eq. (16), $A(E)=H-E S, H$ and $S$ being the Hamiltonian and overlap matrices over the basis set, respectively. Diagonalization is efficiently performed with the inverse iteration procedure. ${ }^{31}$ Partial-wave independent solutions of Eq. (16) are then normalized to incoming wave boundary conditions.

The present LOPT implementation is best described as an independent-particle approach within the effective potential provided by DFT with the ground state density. It is similar in spirit to the static-exchange approximation and may be called a static-DFT approach. The full set of Hamiltonian eigenvectors $\left\{\varphi_{n}^{p \mu}\right\}$,

$$
h_{K S} \varphi_{n}^{p \mu}=\epsilon_{n}^{p} \varphi_{n}^{p \mu}
$$

with bound state boundary conditions, form an effective complete set within the spherical box of radius $R_{\max }^{S C E}$, while the full continuum states satisfy

$$
h_{K S} \Psi_{l h}^{p \mu-}=E \Psi_{l h}^{p \mu-}
$$

where $l h$ count the independent partial wave channels. 


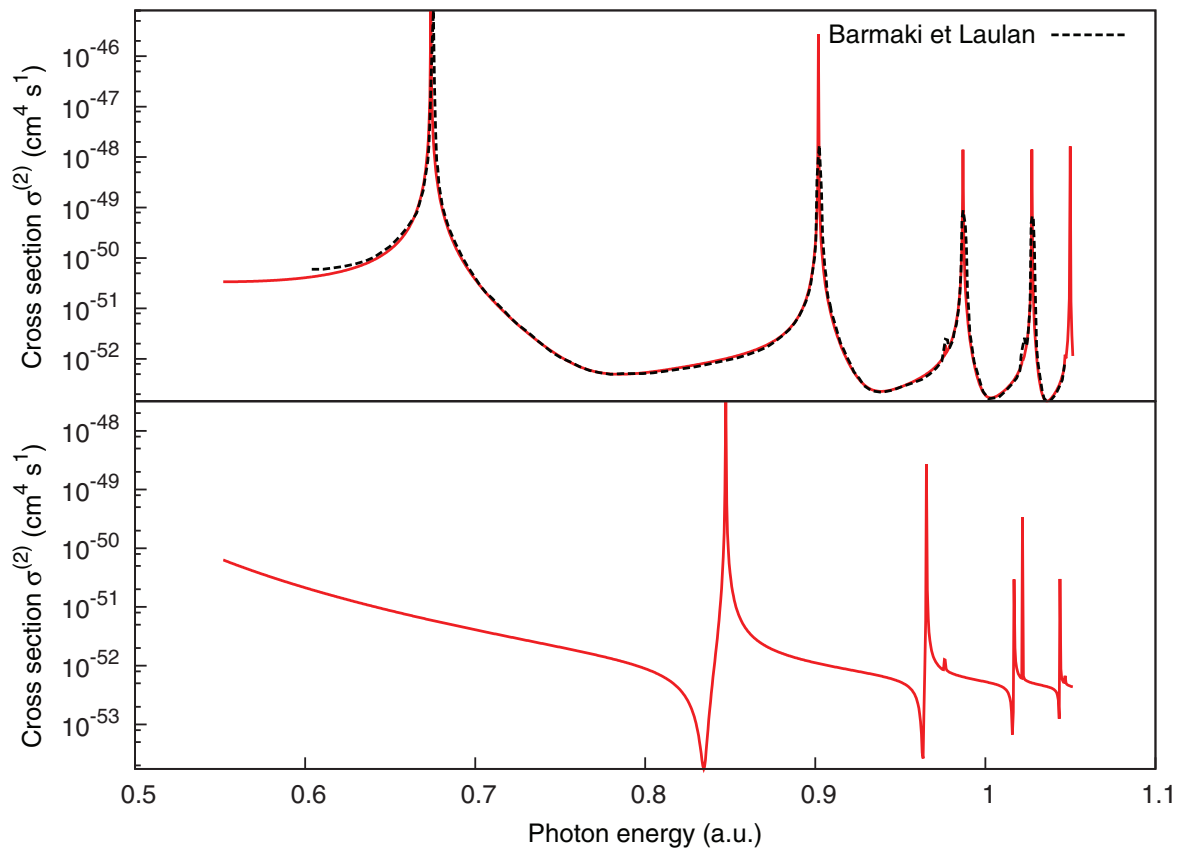

FIG. 1. (Lower panel) Generalized two-photon cross section of $\mathrm{H}_{2}^{+}$for parallel orientation of the molecular axis with respect to the polarization vector of the laser. (Upper panel) Generalized two-photon cross section of $\mathrm{H}_{2}^{+}$for perpendicular orientation of the molecular axis with respect to the polarization vector of the laser. The results of Barmaki and Laulan ${ }^{10}$ are also reported for comparison.

So starting from an initial orbital $\varphi_{i}=\varphi_{i}^{p^{\prime} \mu^{\prime}}$, we can write $M_{i \rightarrow f}^{(N)}=\left\langle f \mid \varphi_{i}^{(N)}\right\rangle$, where

$$
\begin{aligned}
& \varphi_{i}^{(1)}=D \varphi_{i} \\
& \varphi_{i}^{(2)}=D\left(H-\omega_{i}-\omega\right)^{-1} \varphi_{i}^{(1)} \\
& \cdots \\
& \varphi_{i}^{(N)}=D\left(H-\omega_{i}-(N-1) \omega\right)^{-1} \varphi_{i}^{(N-1)} .
\end{aligned}
$$

In the representation of the electronic resolvent given by the complete basis of the Hamiltonian eigenvectors, we have

$$
\varphi_{i}^{(N)}=\sum_{p \mu} \sum_{m}\left|\varphi_{m}^{p \mu}\right| C_{p \mu m, p^{\prime} \mu^{\prime} i}^{(N)}
$$

with

$$
C_{p \mu m, p^{\prime} \mu^{\prime} i}^{(N+1)}(i c)=\sum_{p^{\prime \prime} \mu^{\prime \prime} k} \frac{D_{p \mu m, p^{\prime \prime} \mu^{\prime \prime} k}(\lambda(i c, N)) C_{p^{\prime \prime} \mu^{\prime \prime} k, p^{\prime} \mu^{\prime} i}^{(i c)}}{\omega_{p^{\prime \prime} k}-\omega_{p^{\prime} i}-N \omega}
$$

and

$$
D_{p \mu m, p^{\prime \prime} \mu^{\prime \prime} k}(\lambda(i c, N))=\left\langle\varphi_{m}^{p \mu}|D(\lambda(i c, N))| \varphi_{k}^{p^{\prime \prime} \mu^{\prime \prime}}\right\rangle .
$$

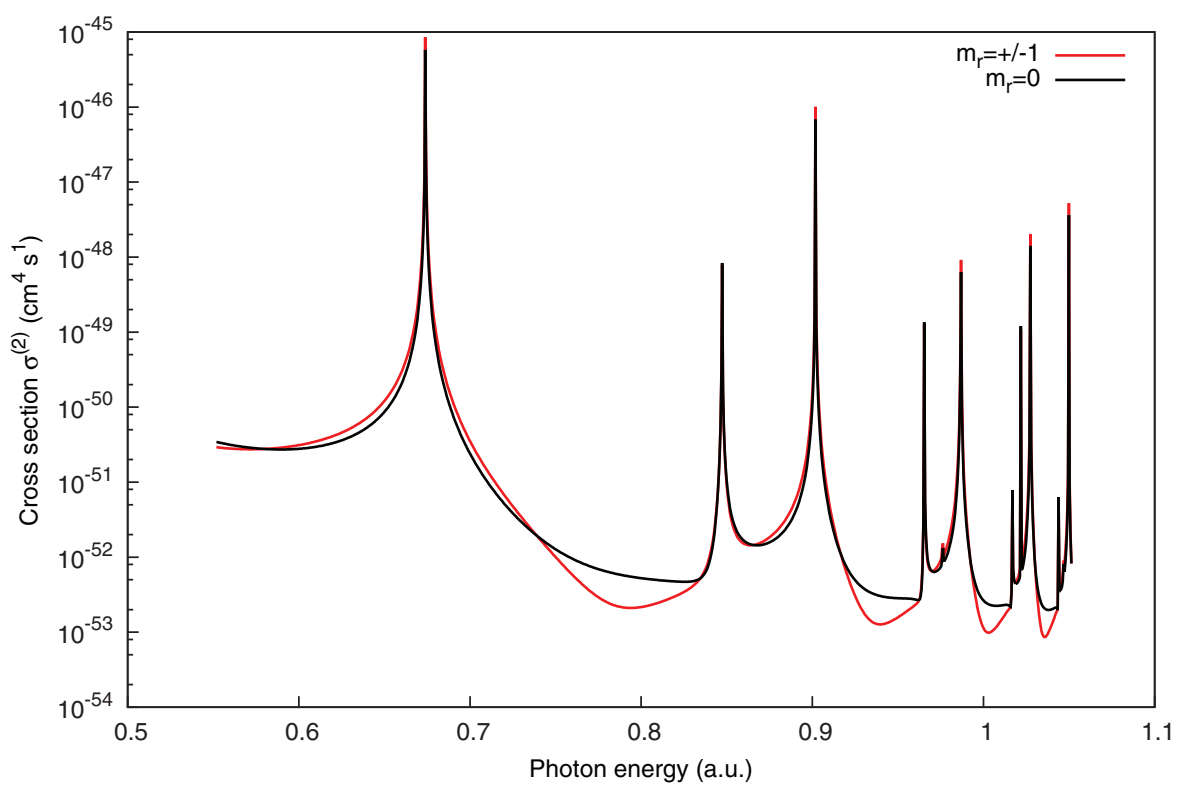

FIG. 2. Rotationally averaged generalized two-photon cross section of $\mathrm{H}_{2}^{+}$for both linearly and circularly polarized photons. 


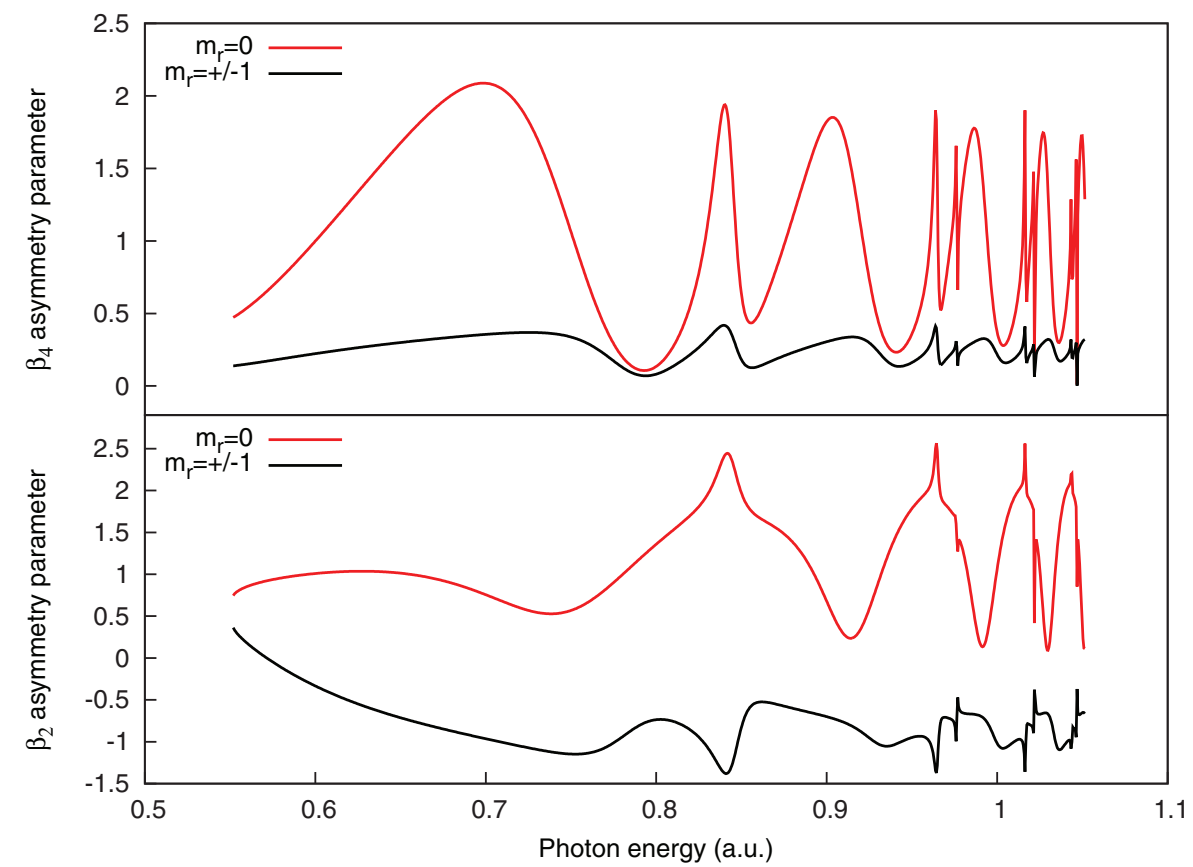

FIG. 3. (Lower panel) Rotationally averaged two-photon asymmetry parameter $\beta_{2}$ of $\mathrm{H}_{2}^{+}$for both linearly and circularly polarized photons. (Upper panel) Rotationally averaged two-photon asymmetry parameter $\beta_{4}$ of $\mathrm{H}_{2}^{+}$for both linearly and circularly polarized photons.

In Eqs. (21) and (22), the integer ic runs over the set of $3^{N}$ combinations of dipole operator components, and $\lambda=x, y$, or $z$.

Therefore, for a given input value of $N$, the expansion coefficients $C_{p \mu m, p^{\prime} \mu^{\prime} i}^{(N)}(i c)$ are obtained after $N-1$ iterations, Eq. (21), starting with

$$
C_{p \mu m, p^{\prime} \mu^{\prime} i}^{(i)}(i c)=\left\langle\varphi_{m}^{p \mu}|D(\lambda(i c, 1))| \varphi_{i}^{p^{\prime} \mu^{\prime}}\right\rangle
$$

for each of the initial orbitals $\varphi_{i}^{p^{\prime} \mu^{\prime}}$.
The procedure is as follows. First, dipole matrix elements between the full set of eigenvectors of the Hamiltonian are calculated and stored on disk. The algorithm takes full advantage of the molecular point group symmetry, so that only those blocks corresponding to symmetry allowed transitions are computed. In the present algorithm, the basis of the eigenvectors is not truncated. For each photoelectron energy in input, dipole matrix elements are read from disk and used to obtain the coefficients $C_{p \mu m, p^{\prime} \mu^{\prime} i}^{(N)}$ of Eq. (21). The scattering wave function, normalized to K-matrix boundary conditions,

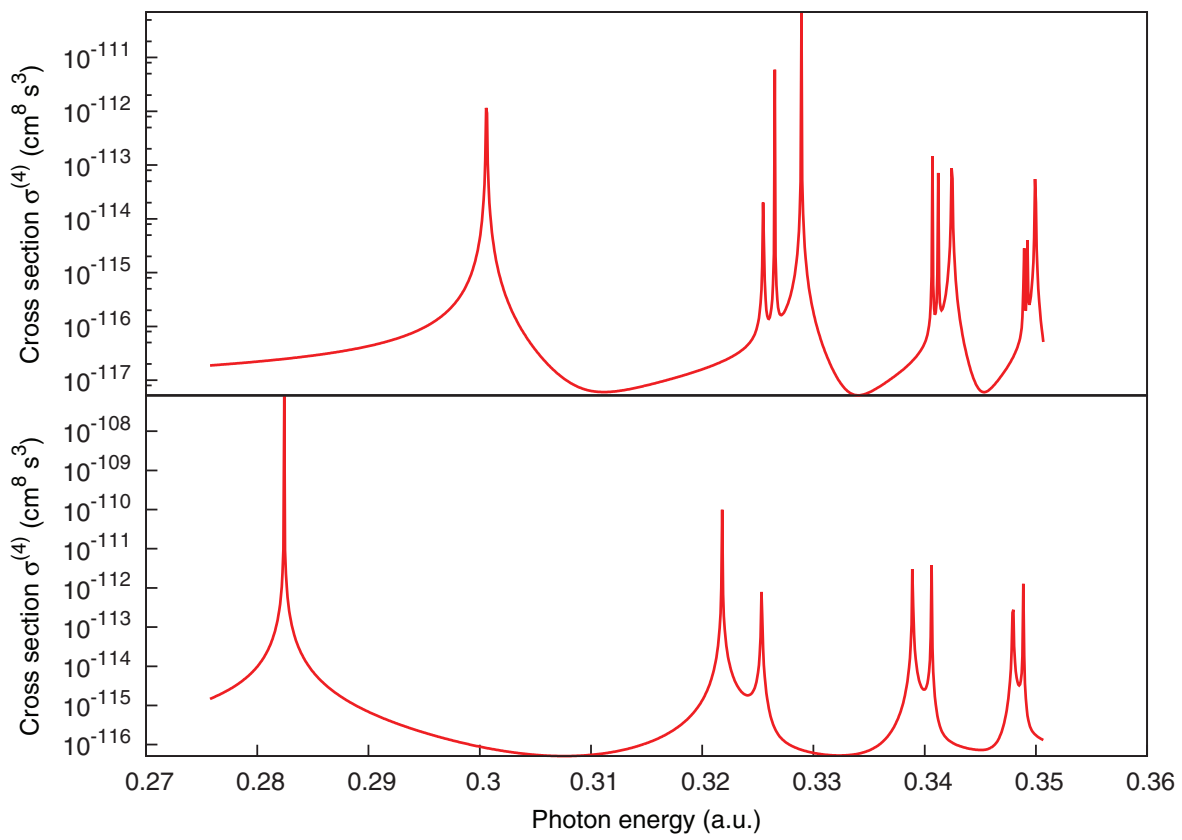

FIG. 4. (Lower panel) Generalized four-photon cross section of $\mathrm{H}_{2}^{+}$for parallel orientation of the molecular axis with respect to the polarization vector of the laser. (Upper panel) Generalized four-photon cross section of $\mathrm{H}_{2}^{+}$for perpendicular orientation of the molecular axis with respect to the polarization vector of the laser. 


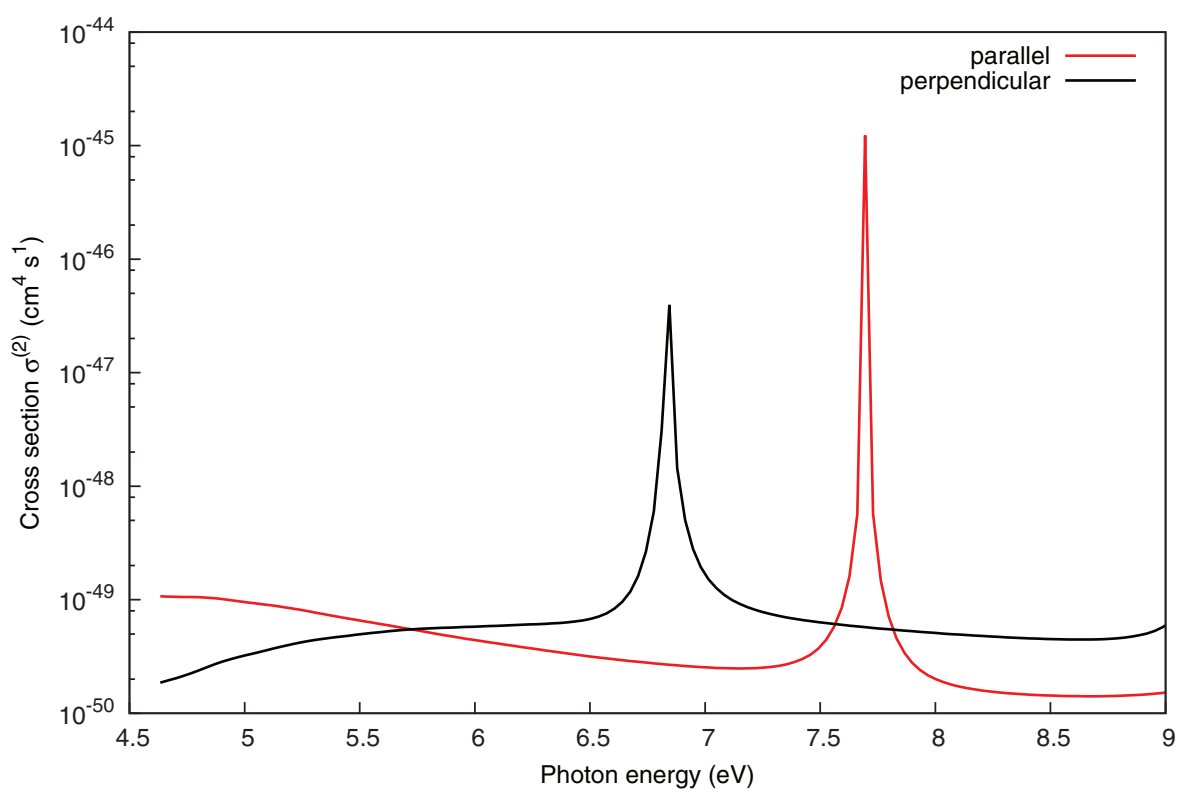

FIG. 5. Generalized two-photon cross section of the HOMO orbital of benzene for both parallel and perpendicular orientation of the molecular plane with respect to the polarization vector of the laser.

is then calculated and the overlap $\left\langle f \mid \varphi_{i}^{(N)}\right\rangle$ is readily obtained. Transition matrix elements are then transformed to S-matrix boundary conditions, and used to calculate the cross sections and angular distribution parameters as detailed in Sec. II.

\section{MULTIPHOTON IONIZATION OF $\mathrm{H}_{2}^{+}$}

An extensive convergence study has been carried out to check the sensitivity of the observables on the parameters specifying the basis set, namely the step size and cutoff of the radial grid of knots, and the number of asymptotic angular momentum channels. We found that the values of $R_{\max }^{S C E}=40$ a.u., $l_{\max }=9$ and a radial step size in the range $0.2-0.4$ a.u. provides convergent results for both the cross sections and angular asymmetry parameters at the internuclear separation of 2.0 a.u. We choose to further increase $R_{\max }^{S C E}$ to 80.0 a.u. and the value of $l_{\max }$ to 15 since this corresponds to a negligible increase of computational effort in the case of the $\mathrm{H}_{2}^{+}$ molecule. Several previous investigations have addressed the calculation of multiphoton ionization cross sections of oriented $\mathrm{H}_{2}^{+}$molecules from linearly polarized light. ${ }^{2,8-13}$ In preliminary studies, we were able to perfectly reproduce the results for $N=2,4$, and 6 photon ionizations quoted by Bachau 9 at selected photon energies and internuclear separations and for a parallel orientation of the molecular axis with respect to the photon polarization vector. In the following, we will

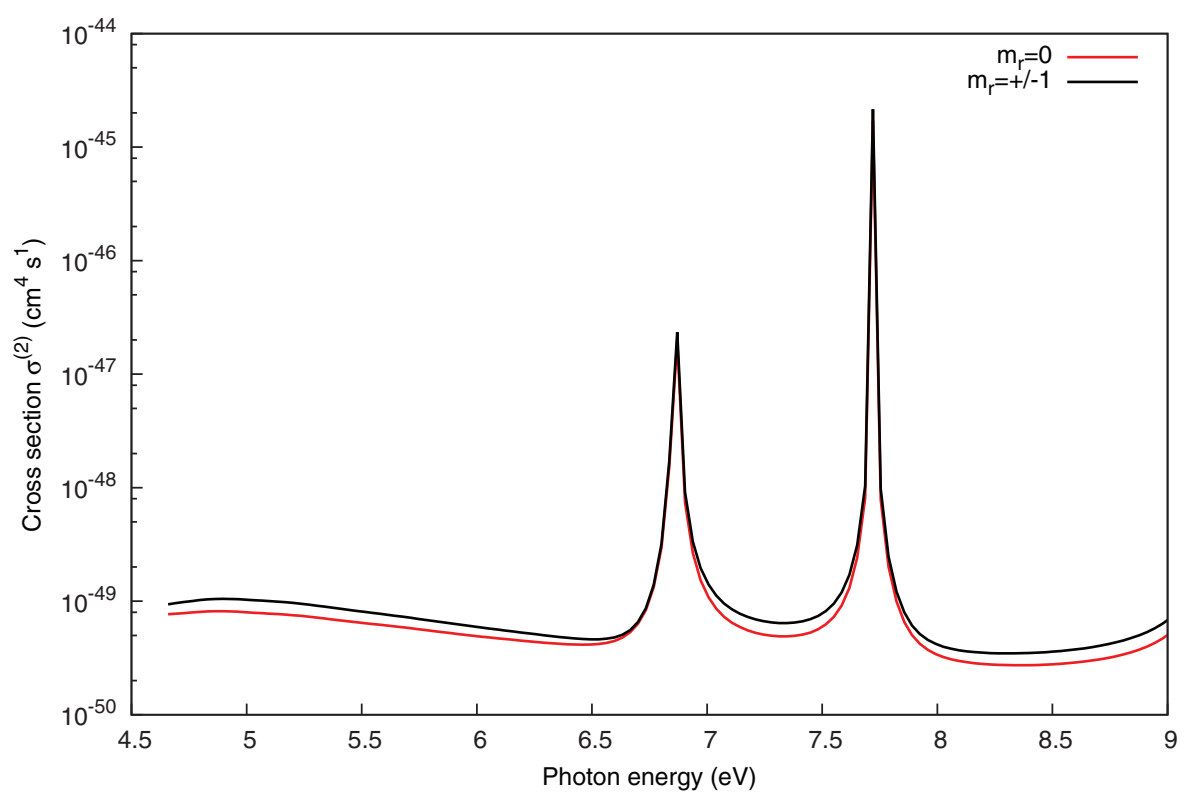

FIG. 6. Rotationally averaged generalized two-photon cross section of the HOMO orbital of benzene for both linearly and circularly polarized photons. 


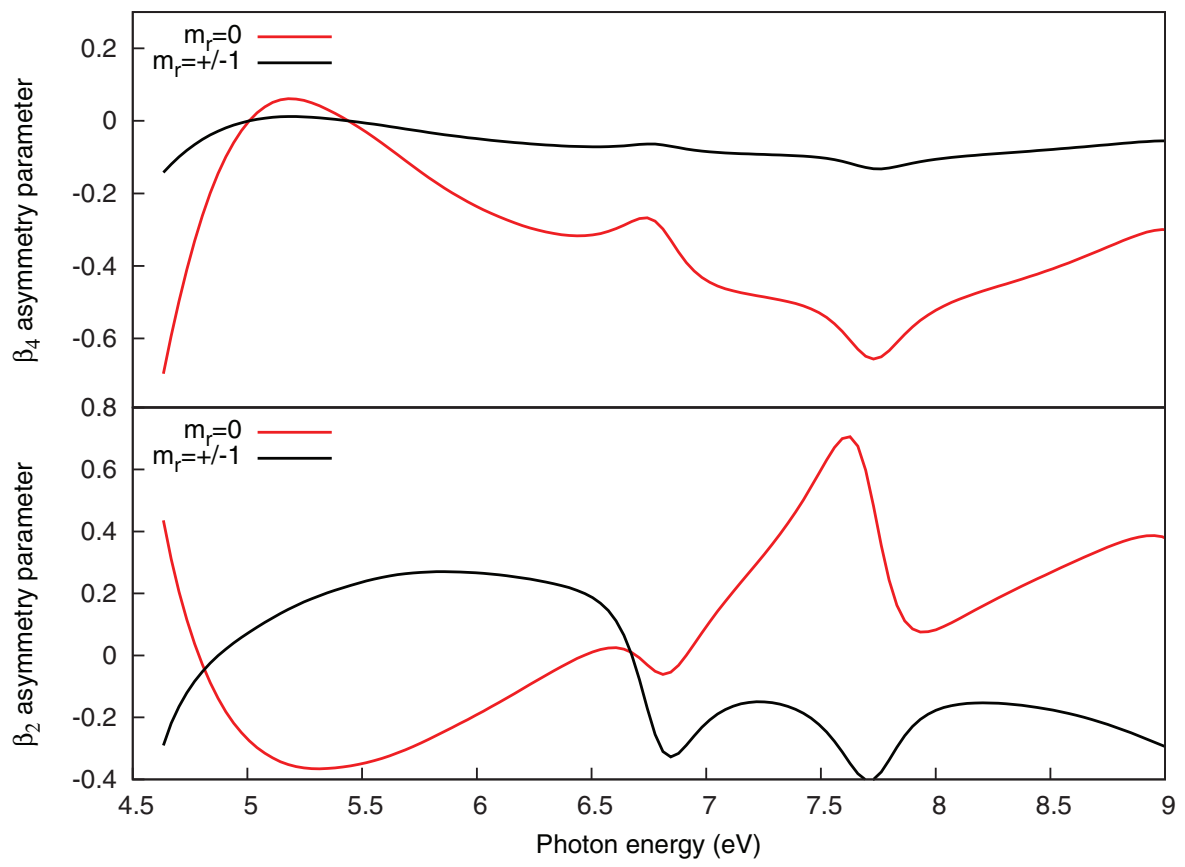

FIG. 7. (Lower panel) Rotationally averaged two-photon asymmetry parameter $\beta_{2}$ of the HOMO orbital of benzene for both linearly and circularly polarized photons. (Upper panel) Rotationally averaged two-photon asymmetry parameter $\beta_{4}$ of the HOMO orbital of benzene for both linearly and circularly polarized photons.

compare our results for $N=2$ and 4 photon ionizations with those presented in their comprehensive study by Barmaki and Laulan, ${ }^{10}$ and for both parallel and perpendicular orientation of the molecular axis with respect to the photon polarization vector.

The two-photon cross section for parallel and perpendicular orientation of the molecular axis with respect to the polarization vector of the field are presented in the lower and upper panel of Figure 1, respectively. In the case of parallel orientation, there is only a single excitation path, namely $\sigma_{g} \rightarrow \sigma_{u}$ $\rightarrow \sigma_{g}$, and the resonant bound states are of $\sigma_{u}$ symmetry. For perpendicular orientation, only transition with $\Delta \lambda= \pm 1$ are allowed, increasing therefore the number of possible excitation paths; the resonances observed in the total cross section profile plotted in the upper panel of Figure 1 correspond to transitions to intermediate states of $\pi_{u}$ symmetry. The classification of resonant features agrees with the one presented by Barmaki and Laulan, ${ }^{10}$ and will not be repeated here. We find an excellent agreement between our data and the highly accurate results presented in Ref. 10, as can be seen from the upper

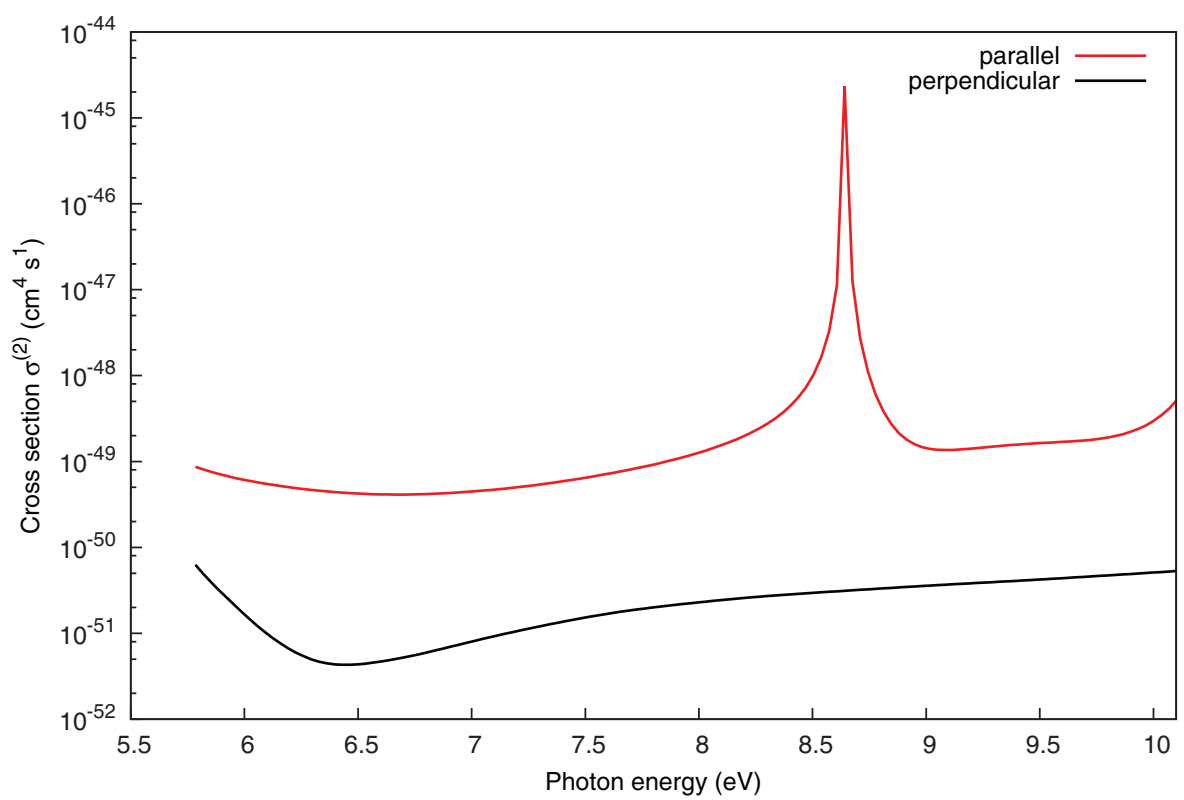

FIG. 8. Generalized two-photon cross section of the HOMO-1 orbital of benzene for both parallel and perpendicular orientation of the molecular plane with respect to the polarization vector of the laser. 


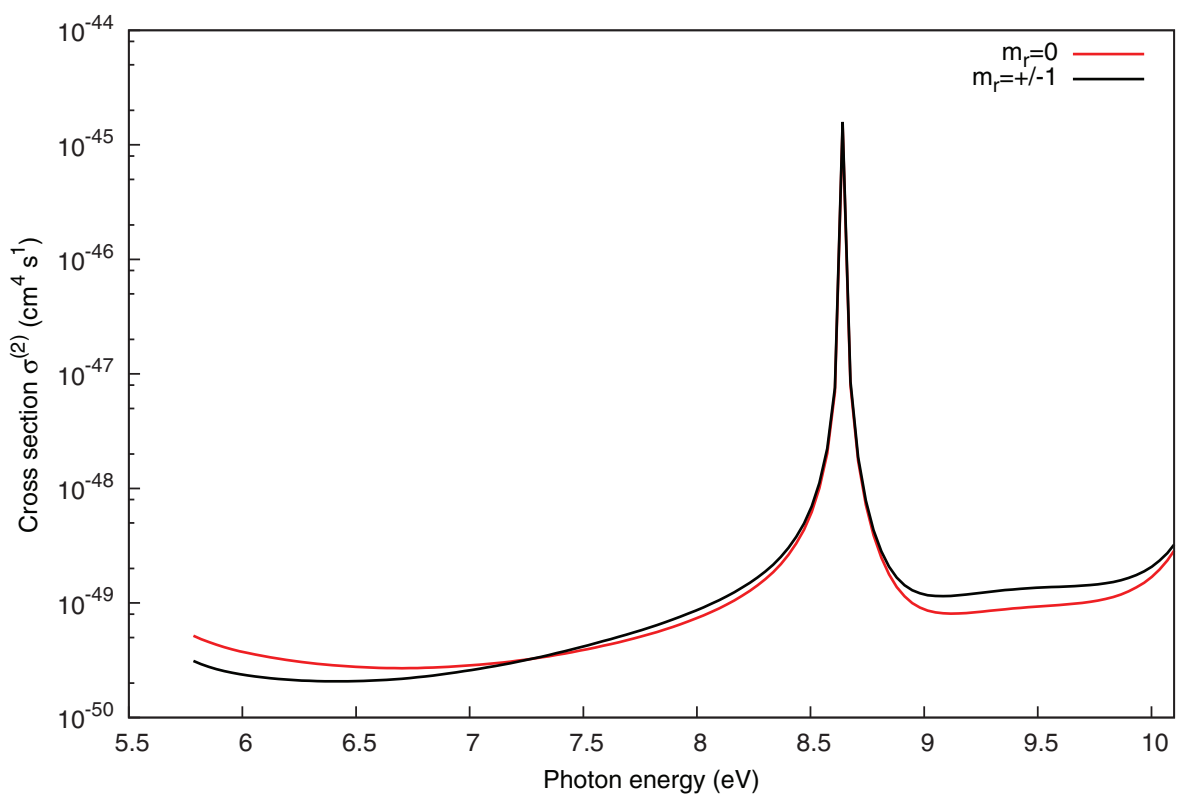

FIG. 9. Rotationally averaged generalized two-photon cross section of the HOMO-1 orbital of benzene for both linearly and circularly polarized photons.

panel of Figure 1. The results of Ref. 10 have been obtained using prolate spheroidal coordinates to take advantage of the symmetry of the two-center one-electron problem. In this respect, the advantage of our approach is that it is completely general and therefore suitable to the calculation of multiphoton cross section of complex polyatomic molecules.

We now turn to the rotationally averaged two-photon cross section and angular distribution parameters, calculated using Eq. (11), for both linear and circular polarization of the laser field. The total cross section is plotted in Figure 2, while the angular distribution parameters $\beta_{2}$ and $\beta_{4}$ are plotted in the lower and upper panel of Figure 3, respectively. To the authors' knowledge there are no such calculations in the literature for the rotationally averaged parameters. We note that both $\beta_{2}$ and $\beta_{4}$ angular distribution parameters display strong modulation in the resonant regions, whose amplitudes are particularly marked in the case of linear polarization compared to the case of circularly polarized photons. Moreover, it does not appear to be a simple relationship between $\beta_{2}$ (and $\beta_{4}$ ) values with linear and circular polarization, so their measurement provides complementary information, at variance with the single photon case. As in the linear regime, $\beta_{1}$ and $\beta_{3}$ are

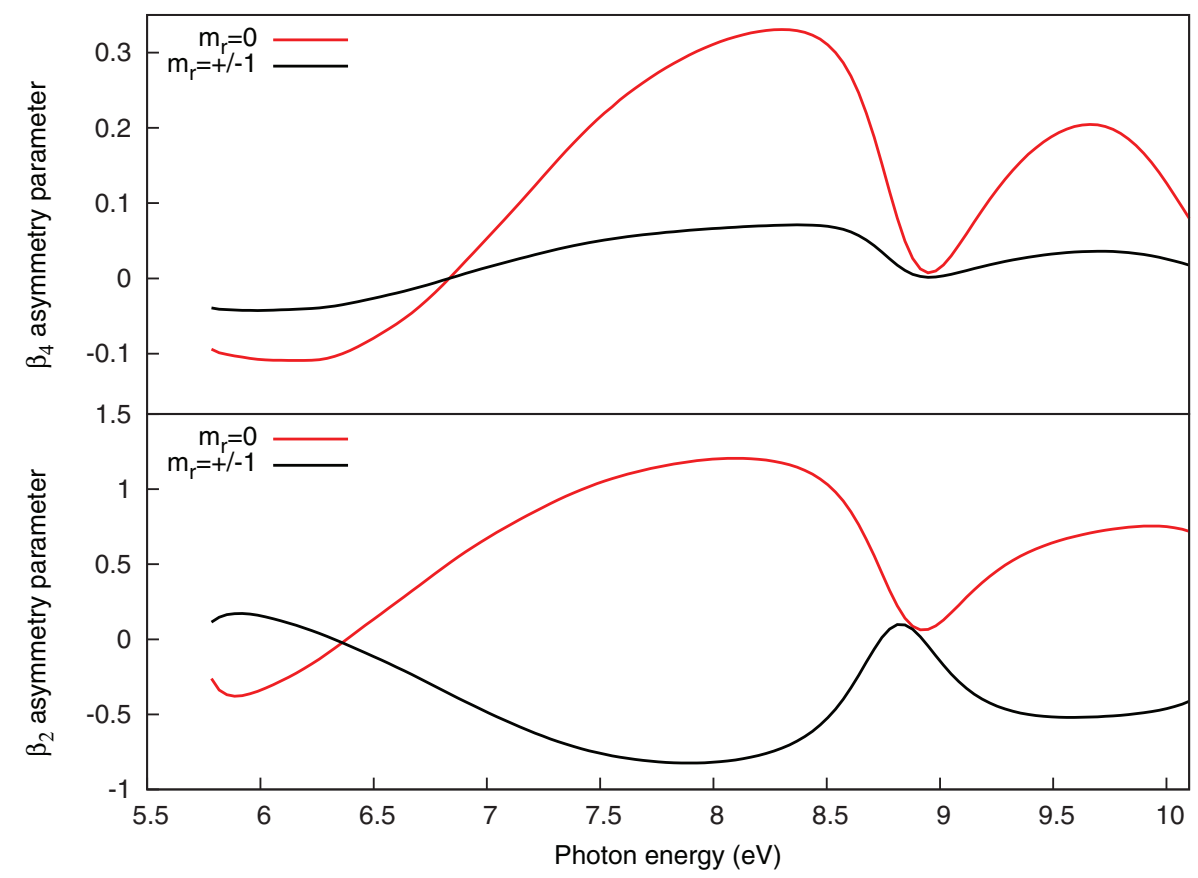

FIG. 10. (Lower panel) Rotationally averaged two-photon asymmetry parameter $\beta_{2}$ of the HOMO-1 orbital of benzene for both linearly and circularly polarized photons. (Upper panel) Rotationally averaged two-photon asymmetry parameter $\beta_{4}$ of the HOMO- 1 orbital of benzene for both linearly and circularly polarized photons. 
zero, unless a chiral system is studied with circular polarized light, in which case they provide a sensitive probe of molecular chirality. ${ }^{23}$

Finally, the generalized four-photons total cross section for oriented $\mathrm{H}_{2}^{+}$molecules is presented in the lower and upper panels of Figure 4 for parallel and perpendicular relative orientation, respectively. Our results compare very well with the ones of Ref. 10.

\section{TWO-PHOTON IONIZATION OF BENZENE}

The experimental equilibrium geometry of benzene $\left(\mathrm{C}_{6} \mathrm{H}_{6}\right)^{32}$ is employed in the calculation of the ground state electron density. Electronic structure calculations at the density functional theory level have been carried out by using the Amsterdam Density Functional (ADF) suite of programs ${ }^{33,34}$ with a double- $\zeta$ plus polarization basis set (DZP) of Slater type orbitals, and the LB94 exchangecorrelation $(x c)$ potential. ${ }^{35}$ All calculations take advantage of the full molecular point-group symmetry $\left(\mathrm{D}_{6 h}\right)$. A preliminary convergence analysis has been carried out to select optimal values of the parameters specifying the radial B-spline basis set, and the number of asymptotic angular momentum channels. Convergent results for both cross sections and angular distribution parameters have been obtained by using a uniform grid of knots with a step size of 0.3 a.u. and radial grid cutoff $\mathrm{R}_{\max }^{S C E}=40$ a.u. The partial wave expansion of the scattering wave function is truncated at a maximum angular momentum quantum number of $l_{\max }=14$. For both HOMO and HOMO-1 ionizations, photoelectron kinetic energies are converted to photon energies by employing the experimental ionization potentials. ${ }^{19}$

We present in Figure 5 the generalized two-photons cross section for the HOMO ionization (orbital $1 \mathrm{e}_{1 g}$ ) and for both parallel and perpendicular orientations of the molecular plane with respect to the polarization vector of the field. Since the molecule is placed in the $\mathrm{x}-\mathrm{y}$ plane, this would correspond to a photon polarization vector along either $\hat{x}$ (or $\hat{y}$ ) and $\hat{z}$ for parallel and perpendicular orientations, respectively. The rotationally averaged generalized cross section for both linearly and circularly polarized photons is plotted in Figure 6. The cross section for perpendicular orientation is dominated by a resonant feature at about $6.8 \mathrm{eV}$ of photon energy, and corresponds to a transition to an intermediate singlet state of $\mathrm{E}_{1 u}$ symmetry. The two-photons cross section for parallel orientation is characterized by a spectral feature at around $7.7 \mathrm{eV}$, which can be ascribed to a one-photon transition to an intermediate singlet state of $\mathrm{A}_{2 u}$ symmetry. These two intermediate resonances are both clearly visible in the rotationally averaged cross section profiles reported in Figure 6. We note that in non-resonant regions, the rotationally averaged cross section for circularly polarized photons is greater than that for linearly polarized radiation, as observed in the case of atomic systems. ${ }^{36,37}$

The rotationally averaged angular asymmetry parameters $\beta_{2}$ and $\beta_{4}$, for both linearly and circularly polarized photons are reported in Figure 7 . The $\beta_{2}$ asymmetry parameter profile displays strong oscillations in resonant regions for both linearly and circularly polarized light. In the near threshold region, the two profiles show marked differences: in the case of circular polarization, the $\beta_{2}$ parameter is characterized by a monotonic increasing behaviour from negative values, at variance with the case of linear polarization, where a steep monotonic decrease from positive values is observed. Strong modulations in the $\beta_{4}$ asymmetry parameter characterize the profile in the case of linear polarization, whereas a comparatively flat profile is predicted for circular polarization.

The generalized two-photon cross section for the HOMO-1 ionization (orbital $3 \mathrm{e}_{2 g}$ ) is reported in Figure 8. Both parallel and perpendicular orientations of the molecular plane with respect to the polarization vector of the field are considered. In the photon energy region investigated, the cross section for perpendicular orientation displays a monotonic increase from about $6.5 \mathrm{eV}$ of photon energy. No resonant features are present. The cross section for parallel orientation is instead characterized by a resonance at about $8.6 \mathrm{eV}$ of photon energy, due to a $3 e_{2 g} \rightarrow 2 e_{1 u}$ one-photon transition. Interestingly, the two-photons ionization cross section for parallel orientation is about two order of magnitude bigger than the cross section for perpendicular orientation. The rotationally averaged cross section is reported in Figure 9 for both linearly and circularly polarized photons.

An analysis of the asymmetry parameter profiles $\beta_{2}$ and $\beta_{4}$ for the HOMO- 1 two-photons ionization, plotted in Figure 10, reveals that both parameters are strongly affected by the presence of the resonant state at $8.6 \mathrm{eV}$ of photon energy. Rather, interesting is the comparison of the computed $\beta_{2}$ profiles for linearly and circularly polarized photons (lower panel of Figure 10): the two profiles display an out-of-phase oscillating behaviour in the whole photon energy interval explored. An oscillating behaviour characterizes also the $\beta_{4}$ profile for linearly polarized light, whereas a rather flat behaviour is predicted in the case of circularly polarized photons.

\section{CONCLUSIONS AND OUTLOOK}

In this work, we present an implementation of the lowest nonvanishing order perturbation theory for the calculation of molecular multiphoton ionization cross sections. Bound and scattering states are calculated in the framework of density functional theory and are expanded in a multicentric symmetry adapted basis set, enabling the application of the formalism to medium-size molecules. General expressions for rotationally averaged ionization cross sections and angular asymmetry parameters have been derived and implemented. Sample calculations for the two- and four-photon ionization of the $\mathrm{H}_{2}^{+}$molecule, and for the two-photons ionization of the HOMO and HOMO-1 orbitals of benzene are reported, demonstrating both the efficiency of the computational scheme and its generality. Multiphoton cross sections for oriented $\mathrm{H}_{2}^{+}$molecules have been compared with accurate data from the literature, and an excellent agreement is found. Moreover, rotationally averaged two-photon cross sections and angular asymmetry parameters for both linear and circular light polarizations have been calculated which were apparently hitherto not considered in detail in the literature.

The present implementation can be extended to a more refined treatment of the many-body problem, such as 
time-dependent DFT or random-phase approximation while preserving the structure of the algorithm and its applicability to extended systems. Future work is planned along these lines in the light of renewed experimental activity in the field due to the availability of the new intense sources provided by FELs and high order harmonic generation sources.

\section{ACKNOWLEDGMENTS}

This research has been supported by TUBITAK of Turkey and CNR of Italy through a bilateral Italy-Turkey project (Grant No. 209T083). Computing resources used in this work were provided by the National Center for High Performance Computing of Turkey (UYBHM) under Grant No. 10972010, and by CINECA of Italy.

${ }^{1}$ H. B. Bebb and A. Gold, Phys. Rev. 143, 1 (1966).

${ }^{2}$ V. A. Davydkin and L. P. Rapoport, J. Phys. B 7, 1101 (1974).

${ }^{3}$ P. Lambropoulos, P. Maragakis, and J. Zhang, Phys. Rep. 305, 203 (1998).

${ }^{4}$ I. Cacelli, V. Carravetta, A. Rizzo, and R. Moccia, J. Chem. Phys. 92, 2883 (1990).

${ }^{5}$ I. Cacelli, V. Carravetta, A. Rizzo, and R. Moccia, J. Chem. Phys. 102, 1230 (1995)

${ }^{6}$ A. Boeglin, B. Fain, and S. H. Lin, J. Chem. Phys. 84, 4838 (1986).

${ }^{7}$ S.-K. Son and S.-I. Chu, Chem. Phys. 366, 91 (2009).

${ }^{8}$ A. Apalategui, A. Saenz, and P. Lambropoulos, J. Phys. B 33, 2791 (2000).

${ }^{9}$ H. Bachau, J. Phys. B 35, 509 (2002).

${ }^{10}$ S. Barmaki and S. Laulan, Can. J. Phys. 88, 1 (2010).

${ }^{11}$ A. Apalategui and A. Saenz, J. Phys. B 35, 1909 (2002).

${ }^{12}$ S. Barmaki, S. Laulan, H. Bachau, and M. Ghalim, J. Phys. B 36, 817 (2003).

${ }^{13}$ M.-G. Baik, M. Pont, and R. Shakeshaft, Phys. Rev. A 54, 1570 (1996).

${ }^{14}$ M. N. R. Ashfold and J. D. Howe, Annu. Rev. Phys. Chem. 45, 57 (1994).
${ }^{15}$ G. Sansone, F. Kelkensberg, J. F. Pérez-Torres, F. Morales, M. F. Kling, W. Siu, O. Ghafur, P. Johnsson, M. Swoboda, E. Benedetti, F. Ferrari, F. Lépine, J. L. Sanz-Vicario, S. Zherebtsov, I. Znakovskaya, A. L. Huillier, M. Y. Ivanov, M. Nisoli, F. Martín, and M. J. J. Vrakking, Nature 465, 763 (2010).

${ }^{16}$ M. Altarelli, Nucl. Instrum. Methods Phys. Res. B 269, 2845 (2011).

${ }^{17}$ M. G. Makris and P. Lambropoulos, Phys. Rev. A 77, 023415 (2008).

${ }^{18}$ D. Toffoli, M. Stener, G. Fronzoni, and P. Decleva, Chem. Phys. 276, 25 (2002).

${ }^{19}$ M. Stener, G. Fronzoni, and P. Decleva, J. Chem. Phys. 122, 234301 (2005).

${ }^{20}$ M. Stener, D. Toffoli, G. Fronzoni, and P. Decleva, Theor. Chem. Acc. 117, 943 (2007).

${ }^{21}$ D. Toffoli, M. Stener, G. Fronzoni, and P. Decleva, Chem. Phys. Lett. 516, 154 (2011).

${ }^{22}$ M. Stener, D. D. Tommaso, G. Fronzoni, P. Decleva, and I. Powis, J. Chem. Phys. 124, 024326 (2006).

${ }^{23}$ D. Catone, M. Stener, P. Decleva, G. Contini, N. Zema, T. Prosperi, V. Feyer, K. C. Prince, and S. Turchini, Phys. Rev. Lett. 108, 083001 (2012).

${ }^{24}$ C. de Boor, A Practical Guide to Splines (Springer, New York, 1978).

${ }^{25}$ H. Bachau, E. Cormier, P. Decleva, J. E. Hansen, and F. Martín, Rep. Prog. Phys. 64, 1815 (2001).

${ }^{26}$ M. Awasthi, Y. V. Vanne, A. Saenz, A. Castro, and P. Decleva, Phys. Rev. A 77, 063403 (2008).

${ }^{27}$ W. Kohn and L. J. Sham, Phys. Rev. A 140, 1133 (1965).

${ }^{28}$ G. Senatore and K. R. Subbaswamy, Phys. Rev. A 34, 3619 (1986).

${ }^{29}$ G. Senatore and K. R. Subbaswamy, Phys. Rev. A 35, 2440 (1987).

${ }^{30}$ N. Chandra, J. Phys. B 20, 3405 (1987).

${ }^{31}$ M. Brosolo and P. Decleva, Chem. Phys. 159, 185 (1992).

${ }^{32}$ G. Herzberg, Electronic Spectra and Electronic Structure of Polyatomic Molecules (Van Nostrand, New York, 1966).

${ }^{33}$ E. Baerends, D. Ellis, and P. Ros, Chem. Phys. 2, 41 (1973).

${ }^{34}$ C. Fonseca-Guerra, J. G. Snijders, G. te Velde, and E. J. Baerends, Theor. Chem. Acc. 99, 391 (1998).

${ }^{35}$ R. van Leeuwen and E. J. Baerends, Phys. Rev. A 49, 2421 (1994).

${ }^{36}$ S. Klarsfeld and A. Maquet, Phys. Rev. Lett. 29, 79 (1972).

${ }^{37}$ B. Ritchie and E. J. McGuire, Phys. Rev. A 24, 2532 (1981). 